\title{
Clima organizacional y desempeño de los docentes en la ULA: Estudio de un caso*
}

\author{
Caligiore Corrales, I rene** \\ Díaz Sosa, J uan Arturo*** \\ ** Profesora Asociada de la Facultad de Medicina. Universidad de Los Andes. Maestría en \\ Gerencia Educativa. E-mail: irenec@ula.ve. Teléfono: 0274-2401111. Extensión 3068. \\ 0274-2449615. Cel. 0414-7444737. Mérida-Venezuela. \\ *** Profesor de Metodología de la Investigación. Maestría en Filosofía. Facultad de \\ Medicina. Universidad de Los Andes. E-mail: jadiaz@ula.ve Teléfono: 0274-2520492. \\ Mérida- Venezuela.
}

\section{Resumen}

Este trabajo es el resultado de un diagnóstico al clima organizacional y el desempeño docente de las escuelas de Enfermería, Nutrición y Medicina de la Facultad de Medicina de la Universidad de Los Andes (ULA) en Venezuela. La investigación se enmarca en la modalidad de proyecto factible, siendo descriptiva y de campo. La población fue de 311 docentes activos y la muestra de 86 individuos, obtenida por muestreo estratificado simple. Se aplicó una encuesta, con una escala de cinco categorías de respuestas. Se realizó el Análisis de la Varianza y la Desviación Estándar de los datos, que reflejan que el puntaje de la valoración global del clima fue de 2.96 en un rango del 1 al 5, ubicándose esto en la categoría en desacuerdo, en cuanto al funcionamiento organizacional de la facultad por ser mecánica e ineficiente. No hubo diferencias significativas entre Medicina y Enfermería referente a la variable Desempeño Docente, aunque si con Nutrición, lo que podría estar relacionado con el estilo gerencial y la calidad de las relaciones interpersonales. Se concluye sobre la necesidad de adecuar la estructura organizativa a las funciones sustantivas de la universidad, facilitando la coordinación y la ejecución de las decisiones.

Palabras clave: Clima organizacional, gestión, desempeño académico, docente universitario.

\section{Organizational Climate and Performance of ULA Teachers: A Case Study}

\section{Abstract}


This paper is the result of a organizational climate and teacher performance diagnosis undertaken in the Schools of Nursing, Nutrition and Medicine at the University of Los Andes (ULA) in Venezuela The research was carried out as a factibility project study, was descriptive, and field based. The population was 311 active teachers and a sample of 86 was selected through simple stratified sampling techniques. A questionnaire was applied with answers based on a five category scale. Analysis of Variance and Standard Deviation was applied to the data which indicated a global evaluation of 2.96 on a scale of 1 to 5 , which corresponded to the answer "disagree" in relation to the organizational functioning of the faculty due to its being mechanical and inefficient. There were no significant differences between the Schools of Medicine and Nursing in terms of teacher performance, but there was a difference in relation to the School of Nutrition which could be related to the management style and the quality of interpersonal relationships. The conclusion was the need to improve the organizational structure of the basic functions of the university, faciliting in this manner the coordination and execution of decisions.

Key words: Organizational climate, management, academic performance, university teachers.

Recibido: 02-11-20. Aceptado: 03-10-09

\section{I ntroducción}

Una organización es un grupo de personas con responsabilidades específicas, que actúan juntas para el logro de un propósito específico determinado por la organización. Todas las organizaciones tienen un propósito, una estructura y una colectividad de personas. La universidad como institución educativa es una organización y está conformada por un grupo de elementos interrelacionados entre sí, tales como: la estructura organizacional, los procesos que ocurren dentro de ella y la conducta de los grupos e individuos. La interacción de los componentes antes mencionados produce patrones de relación variados y específicos que encajan en lo que se ha denominado Clima Organizacional.

Este es un fenómeno macro-organizacional que ha sido estudiado en los últimos tiempos por la escuela humanista administrativa, donde los investigadores comienzan sus indagaciones preguntándoles a los miembros de las organizaciones cómo lo perciben. En suma, el clima organizacional es el ambiente interno de una organización que no es físicamente palpable, pero sí se siente. 
Según refiere Goncalves (1999:2), "el clima organizacional es un componente multidimensional de elementos que pueden descomponerse en términos de estructuras organizacionales, tamaño de la organización, modos de comunicación, estilos de liderazgo de la dirección, entre otros". Todos estos elementos se suman para formar un clima particular dotado de sus propias características, que presenta en cierto modo, la personalidad de una organización e influye en el comportamiento de un individuo en su trabajo.

La importancia de este enfoque reside en el hecho que el comportamiento de un trabajador no es una resultante de los factores organizacionales existentes, sino que depende de las percepciones que él tenga de estos factores. Sin embargo, estas percepciones dependen en buena medida de las actividades, interacciones y otra serie de experiencias de cada miembro con la organización. De ahí que el Clima Organizacional refleja la interacción entre las características personales y organizacionales (Schneider y Hall, citados por Goncalves, 1999:3).

En este sentido, se destacan los resultados del estudio realizados por George y Bishop (citados por Dessler 1991:188), quienes refieren que la estructura organizacional (que incluye la división del trabajo, los patrones de comunicación y los procedimientos) tiene gran efecto sobre la manera como los empleados visualizan el clima de la organización. El grado en que la empresa sea mecánica o burocrática influye en esa percepción, señalando, además, que los sistemas educativos muy burocráticos (altamente estructurados y centralizados) se veían como climas cerrados, restrictivos.

En este orden de ideas, se plantea la actual situación de la Facultad de Medicina de la U.L.A. La Universidad de Los Andes es una universidad nacional autónoma, financiada por el estado en un $90 \%$, cuyo régimen de estudios en la mayoría de las carreras es semestral y en otras es de anualidad. Su estructura académica está constituída por diez Facultades y dos Núcleos; estos a su vez se subdividen en Escuelas y estas en Departamentos, los que a su vez están conformados por Cátedras. También cuenta con institutos, centros y laboratorios de investigación. La máxima autoridad está representada por el Rector, quien preside el Consejo Universitario (Universidad de Los Andes, 1995: 2).

De lo anterior se deduce que esta organización universitaria está basada en la burocracia, la cual representa una estructura jerárquica piramidal y formal establecida (cadena de mando), con actividades y objetivos determinados, especialización de las tareas, además de reglamentos bien definidos (Stoner, Hughes y Tight, 1996: 89). Sin embargo, hay que señalar que este modelo de administración, implementado desde hace 
mucho tiempo, ha conducido a esta universidad lentitud de las actividades, rutinización y muchas veces ineficiencia de las mismas, conllevando a un clima impersonal y cerrado.

La Facultad de Medicina está compuesta por tres escuelas: Enfermería, Medicina y Nutrición, respectivamente; donde existe una estructura organizativa vertical y rígida integrada por cuatro niveles claramente definidos como son: el Consejo de Facultad, el Consejo de Escuela, el Consejo de Departamento y las cátedras, presididos respectivamente por el Decano, el Director, el Jefe de Departamentos y los Jefes de Cátedras (estas pueden estar integradas por dos o más profesores).

En el primer nivel se conocen y deciden todas las situaciones inherentes a las cátedras de cada escuela y sus profesores, las cuales muchas veces no son dadas a conocer de manera efectiva a los niveles jerárquicos inferiores generando una situación de desinformación y tergiversación de la misma. Además, existen grupos (que mantienen una estrecha relación con esta instancia de decisión), que a su vez ejercen el poder de forma autocrática impidiendo el planteamiento de otras alternativas y conllevando a situaciones conflictivas (confusión, desconfianza y rechazo) entre los miembros de esta organización universitaria. Por otra parte, se percibe un trato desigual hacia los profesores de las Escuelas de Enfermería y Nutrición (pues son minoría con relación al número de profesores y alumnos de la Escuela de Medicina) ya que el Consejo de Facultad está integrado por el $90 \%$ de profesores de la Escuela de Medicina, aunque el Director de cada escuela es miembro del mismo; por lo que muchas de las decisiones que se toman y que afectan el funcionamiento de las escuelas de Enfermería y Nutrición son decididas por una mayoría que no está enterada de las situaciones que afectan a las mismas. En consecuencia, dependerá del liderazgo de cada director para que logre demostrar la importancia que merecen las situaciones particulares planteadas por ellos y se genere así una justa y efectiva decisión a los asuntos planteados en el seno del Consejo de esta Facultad.

De lo anteriormente descrito se aprecia la existencia de algunos de estos factores (estructura organizacional, estilo de liderazgo, sistema de recompensas, tipo de relaciones interpersonales, normas y reglas existentes) que estarían contribuyendo a que en esta facultad, se perciba cierto grado de apatía e insatisfacción por parte de los miembros de la organización, quienes manifiestan tener expectativas desfavorables en lo que respecta a sus aspiraciones profesionales, académicas y sociales. En consecuencia, esta situación podría estar influyendo en el desempeño de los docentes de cada escuela de esta facultad, generando un comportamiento manifiesto de los mismos, producto de las percepciones que filtran la realidad y condicionan los niveles de motivación y rendimiento profesional. 
Por otra parte, no se puede ignorar el hecho que también el estudiante pudiera ser sensible a la influencia intangible del clima de la institución.

El propósito de esta investigación fue realizar un diagnostico sobre el clima organizacional existente en las escuelas de Enfermería, Nutrición y Medicina de la Facultad de Medicina de la Universidad de Los Andes, a fin de determinar el nivel de desempeño de los mismos y elaborar una propuesta de gestión para optimizar la situación existente.

\section{Antecedentes}

En Venezuela, el estudio del clima organizacional surgió a partir de la década de los 80, por el interés de conocer los factores organizacionales que estaban influyendo en el servicio prestado por las diferentes organizaciones. Se destaca el estudio realizado por Pérez y Sanabria (1997) sobre el Clima organizacional en el Decanato de Ingeniería Agronómica de la Universidad Centro- occidental Lisandro Alvarado, cuyo propósito fue diagnosticar según la percepción de los docentes, directivos y administrativos los factores relacionados con el comportamiento individual y grupal, la motivación, el liderazgo, y los procesos organizacionales, comunicación, toma de decisiones y rendimiento. Consistió en una investigación descriptiva en la cual participaron 82 docentes, 15 directivos y 62 administrativos. El diagnóstico se realizó mediante la aplicación individual de un cuestionario elaborado para tal fin. Se encontró que predominaba un clima autoritario benevolente, con ciertos rasgos del sistema consultivo, caracterizado por: ocasionalmente se aceptan ideas y opiniones de los subordinados, estilos de liderazgos no definidos, algunas veces rígidos ya que los directivos asumen la mayor responsabilidad en la toma de decisiones, comunicación predominantemente descendente y unidireccional, usada para informar de las decisiones tomadas aunque tardíamente. Se señalaron acciones a seguir para lograr un cambio en aquellos elementos del clima considerados desfavorables, según la percepción del personal entrevistado.

De igual manera, Solla (1998) refiere que realizó un estudio sobre el clima organizacional y su relación con la calidad del servicio prestado por el Colegio Universitario de Administración y Mercadeo, Extensión Puerto Cabello en Venezuela, el cual tuvo como propósito analizar la relación que existe entre el clima organizacional y la calidad del servicio prestado por esta organización. A una muestra de 344 sujetos, conformados por un directivo, 25 docentes, 10 administrativos y 308 alumnos a los que se les aplicaron dos cuestionarios, encontrándose en las respuestas de los individuos de estos estratos, discrepancias marcadas al opinar sobre la responsabilidad de la gerencia, el conocimiento de los objetivos y normativa de la institución, los recursos didácticos utilizados, la 
existencia del material de apoyo, el costo de los servicios y el recurso involucrado. Por ello recomienda que todo proceso que involucre el clima organizacional y la calidad del servicio debe estar previamente planificado, revisado y evaluado para que este permita orientarse hacia los niveles de excelencia.

Así mismo, López et al (1999) realizaron un estudio cuyo propósito fue conocer el clima laboral de los profesionales que trabajan en los establecimientos de atención primaria (ambulatorios) de la región de Murcia (Colombia) y analizar su evolución transcurrido un año de su creación. Los resultados reflejan que la valoración global del ambiente de trabajo entre los profesionales, transcu- rrido un año desde la creación de estos establecimientos es muy elevada, con expectativas favorables. La tendencia global es hacia un deterioro del clima organizacional lo que permite deducir que en el ambiente de trabajo los recursos humanos y algunos elementos organizacionales (motivación y participación) son variables que inciden en la calidad de los servicios y en el cumplimiento de los objetivos propuestos (Menárquez y Saturno, 1999).

De igual manera, Feliú (2000), refiere la evaluación del clima organizacional y la satisfacción de los empleados de la compañía Cigarrera Bigott de Venezuela. Los aspectos que resultaron mejor ponderados por los empleados en cuanto al clima organizacional fueron la definición de visión, misión y valores de la empresa, así como la concordancia entre esto y los objetivos de sus empleados. Los items peor ponderados fueron el conocimiento por parte de los empleados de los avances y logros de otras áreas de la empresa, lo que guarda relación con el funcionamiento de los canales de comunicación, el rol que ejercen los supervisores y la valoración del desempeño de los trabajadores. En total, la media para el clima organizacional fue de 4,42 puntos en un rango de puntuación del 1 al 5.

\section{Metodología}

Este estudio se enmarca en la modalidad de Proyecto factible, el cual ha sido definido por la UPEL (1996:7) de la siguiente manera: “consiste en la investigación, elaboración y desarrollo de una propuesta de un modelo operativo viable para solucionar problemas, requerimientos o necesidades de organizaciones o grupos sociales; puede referirse a la formulación de políticas, programas, tecnologías, métodos o procesos. El proyecto debe tener el apoyo en una investigación de tipo documental, de campo o un diseño que incluya ambas modalidades".

La población estuvo conformada por 311 docentes activos que estaban adscritos a la 
Facultad de Medicina, en este grupo no se incluyeron los docentes de las extensiones que están fuera del estado Mérida. Estos datos fueron obtenidos en el Departamento de Administración del Decanato de Medicina de la Universidad de Los Andes.

La muestra estuvo conformada por 86 docentes activos. De este grupo se tomaron docentes de cada una de las tres escuelas que conforman la Facultad de Medicina, aplicando el Muestreo Estratificado Simple. Para tal fin, se utilizó la nómina de los docentes adscritos a cada escuela de esta Facultad (Tabla 1).

La Muestra Probabilística se calculó según lo planteado por Sierra (citado por la UPEL, 1998:272)

$$
\begin{aligned}
& n=\frac{4 \cdot p \cdot q \cdot N}{E^{2}(N-1)+4 . p \cdot q} \\
& \frac{4.50 .50 .311}{(02)^{2}(310)+4.50 .50}=\frac{3.110 .000}{41.000}=86
\end{aligned}
$$

La recolección de los datos se realizó a través de un cuestionario. El mismo tenía 32 items, estructurados con una escala tipo Lickert, de cinco categorías de respuestas y con un rango del 1 al 5, el cual fue validado mediante juicio de expertos.

La confiabilidad del instrumento se obtuvo de los resultados de la prueba piloto. En este caso, el instrumento se le aplicó a doce docentes, profesionales de las ciencias de la salud, adscritos a la Facultad de Farmacia y Odontología de la Universidad de Los Andes, por tener características similares a los docentes de la Facultad de Medicina. Posteriormente, se calculó el Coeficiente Alfa de Cronbach, el cual dio como resultado: 0,896.

\section{Tabla 1}

ULA-Facultad de Medicina Distribución de la Muestra por Estratos

\begin{tabular}{lccc}
\hline Estrato & Población & Muestra & $\%$ \\
\hline Escuela de Enfermeria & 34 & 19 & 54 \\
Escuela de Nutrición & 57 & 14 & 24 \\
Escuela de Medicina & 220 & 53 & 22 \\
Total & 311 & 86 & 100 \\
\hline
\end{tabular}

Fuente: Universidad de Los Andes (2001).

\section{Resultados del diagnóstico}


La encuesta aplicada a los docentes de las escuelas de Enfermería, Medicina y Nutrición, estuvo conformada por 32 preguntas, en la que cada pregunta tenía 5 alternativas de respuestas según la escala tipo Likert, donde el entrevistado debía seleccionar sólo una respuesta a cada pregunta. De acuerdo a esa escala se agruparon las respuestas en tres categorías, para lo cual se construyeron los siguientes intervalos de puntuaciones para analizar el Clima Laboral y el Desempeño Docente:

$$
\begin{aligned}
& L_{i}----L_{s} \text { Ca tegoría } \\
& 32---74 \text { Deficiente } \\
& 75--117 \text { Regular } \\
& 118---160 \text { Excelente }
\end{aligned}
$$

A fin de lograr los objetivos planteados en este estudio, las variables operacionalizadas fueron: Clima Organizacional y Desempeño Docente. Para ello se consideró el puntaje mínimo y máximo que se le podían asignar a cada uno de los items de cada indicador para su ubicación en las categorías de la escala (Deficiente, Regular y Excelente), cuyos resultados son los siguientes:

Clima Organizacional, cuyos valores van desde 24-56 puntos correspondiendo a la categoría Deficiente; 57-88 puntos que corresponde a la categoría Regular; 89-120 puntos que corresponde a Excelente.

Desempeño Docente, cuyos valores van desde 8-19 puntos correspondiendo a la categoría Deficiente; 20-30 puntos corresponde a la categoría Regular; 31-40 puntos corresponde a Excelente.

Estas variables, a su vez se clasificaron y analizaron por dimensiones, dividiéndose la variable Clima

Organizacional en las siguientes dimensiones:

Funcionamiento Organizacional, Conformada por tres indicadores, cuyos valores van desde 6-14 puntos, considerándose como Deficiente; 15-22 puntos se consideran Regular; 23-30 puntos se consideran como Excelente.

Estructura Organizacional, conformada por tres indicadores, cuyos valores se ubican desde 6-14 puntos, se consideran como Deficiente; 15-22 puntos se consideran como 
Regular; 23-30 puntos se considera Excelente.

Proceso de Toma de Decisiones, conformada por un indicador cuyos valores van desde 2-4 puntos, los cual se considera como Deficiente; 5-8 puntos se considera como Regular; 9-10 puntos se considera como Excelente.

Tipo de Comunicación, conformada por dos indicadores cuyos valores van desde 4-10 puntos, lo cual se considera como Deficiente; 11-15 puntos se considera como Regular; 16-20 puntos se considera como Excelente.

Motivación al Logro, conformada por tres indicadores cuyos valores van desde 6-14 puntos, lo cual se considera como Deficiente; 15-22 puntos se considera como Regular; 23-30 puntos se considera Excelente.

La variable Desempeño docente tuvo una sola dimensión que se denominó: Praxis Educativa, conformada por cuatro indicadores, cuyos valores van desde 8-19 puntos lo cual se considera como Deficiente; 20-30 puntos que se considera como Regular; 31-40 puntos que se considera como Excelente.

Cuando se compararon las tres escuelas de la Facultad de Medicina de la ULA (Tabla 2), a fin de ubicar las respuestas de acuerdo a las categorías anteriores, se obtuvo el siguiente resultado:

\section{Tabla 2}

ULA-Facultad de Medicina. Distribución de respuestas de los docentes sobre el clima laboral y el desempeño docente

\begin{tabular}{lrrrrrrr}
\hline Escuela & $N$ & Media & $\begin{array}{c}\text { Desviación } \\
\text { Estándar }\end{array}$ & $\begin{array}{r}95 \% \\
\text { L. Confianza Intervalos } \\
\text { depara la media } \\
\text { L. Inferior }\end{array}$ & L. Superior & Mimo & Máximo \\
\hline Nutrición & 14 & 102,2143 & 27,0844 & 86.5762 & 17,8524 & 57,00 & 136,00 \\
Medicina & 53 & 108,6981 & 21,9050 & 102.6603 & 114,7359 & 45,00 & 151,00 \\
Enfermería & 19 & 101,3158 & 22,8402 & 90,3072 & 112,3244 & 59,00 & 140,00 \\
\hline
\end{tabular}

Fuente: Encuesta para este trabajo.

N: Tamaño de la muestra.

Se pudo observar que la puntuación media de las tres escuelas se ubicó en el segundo intervalo de la escala que corresponde a la categoría regular y al realizar el análisis de la varianza se observa que no existen diferencias significativas entre las tres escuelas en cuanto a las variables Clima Laboral y Desempeño Docente. De esto se deduce que los docentes de estas escuelas tienen una tendencia a valorar el clima organizacional de la 
Facultad de Medicina de forma débil y negativo, ya que lo sitúan en el intervalo de la escala que muestra desacuerdo con las condiciones actuales de los siguientes procesos: estilo gerencial, supervisión, relaciones interpersonales estructura organizacional, proceso de toma de decisiones, motivación y en la comunicación.

En cuanto a la Escuela de Nutrición se observa (Tabla 3), que en la dimensión Funcionamiento Organizacional presenta un valor de la Media de 17.7 puntos que corresponde a la categoría Regular, de igual manera se ubican las dimensiones: Estructura Organizacional, Tipo de comunicación, Motivación al Logro. Sin embargo, a diferencia de estas, se destaca que el valor obtenido en la dimensión Proceso de Toma de Decisiones se ubicó en la categoría Deficiente. Esto podría estar relacionado con la forma como este proceso se lleva cabo dentro de esta escuela, generando tal vez, frecuentes discrepancias o desacuerdos entre directivos y docentes.

Tabla 3

ULA-Escuela de Nutrición. Distribución de las respuestas según las dimensiones estudiadas

\begin{tabular}{lccccc}
\hline \multicolumn{1}{c}{ Dimensión } & N & Minimo & Máximo & Media & Desviación Estándar \\
\hline $\begin{array}{l}\text { Funcionamiento } \\
\text { Organizacional }\end{array}$ & 14 & 8,00 & 27,00 & 17,7143 & 6,2441 \\
$\begin{array}{l}\text { Estructura Organizacional } \\
\text { Proceso de Toma }\end{array}$ & 14 & 6,00 & 25,00 & 15,5714 & 5,5291 \\
de Decisiones & 14 & 2,00 & 8,00 & 4,6429 & 2,0979 \\
Tipo de comunicación & 14 & 5,00 & 18,00 & 11,6429 & 4,3608 \\
$\begin{array}{l}\text { Motivación al Logro } \\
\text { Praxis Educativa }\end{array}$ & 14 & 11,00 & 29,00 & 22,0000 & 6,3002 \\
$\begin{array}{l}\text { Variable Clima } \\
\text { Organizacional }\end{array}$ & 14 & 17,00 & 30,00 & 24,0714 & 3,9897 \\
$\begin{array}{l}\text { Variable Desempeño } \\
\text { Docente }\end{array}$ & 14 & 32,00 & 100,00 & 71,5714 & 22,0828 \\
\hline
\end{tabular}

Fuente: Encuesta para este trabajo.

N: Tamaño de la muestra.

En cuanto a la variable: Clima Organizacional, también se observa, que esta obtuvo un valor que la ubica en la categoría Regular, igual que el valor obtenido en la variable Desempeño Docente. Estos resultados permiten deducir la forma como está siendo percibido el clima en la Escuela de Nutrición por los miembros del cuerpo profesoral. Lo que trae consigo, afirmar que en la misma se estarían generando situaciones no ideales, que estarían limitando el desarrollo del trabajo y la productividad de los docentes (Tabla 4). 
Tabla 4

ULA-Escuela de Nutrición

Distribución de las respuestas según las variables estudiadas

\begin{tabular}{lccccc}
\hline Variable & N & Mínimo & Máximo & Media & Desviación Estándar \\
\hline Organizacional & 14 & 32,00 & 100,00 & 71,5714 & 22,0828 \\
$\begin{array}{l}\text { Desempeño } \\
\text { decente }\end{array}$ & 14 & 19,00 & 40,00 & 30,3429 & 5,9305 \\
\hline
\end{tabular}

Fuente: Para este trabajo.

N: Tamano de la muestra.

Al respecto, es importante destacar la forma tan negativa como que fue valorado el proceso de Toma de Decisiones que se lleva a cabo dentro de esta escuela, sobre todo en lo referente a la manera en que los directivos plantean y resuelven los problemas institucionales, alejados del personal docente y sin consenso, lo que genera que las mismas no sean difundidas oportunamente. Por otra parte, llama la atención que los docentes de esta escuela también señalan su desacuerdo con el estilo gerencial existente porque consideran que el mismo no favorece el trabajo productivo.

En cuanto a los resultados obtenidos en la Escuela de Medicina de esta Facultad (Tablas 5 y 6), se observa que las dimensiones: Funcionamiento Organizacional, Estructura Organizacional, Tipo de Comunicación, que pertenecen a la variable Clima Organizacional, obtuvieron valores que se sitúan en la categoría Regular. No así, el Proceso de Toma de Decisiones que fue considerado como Deficiente, al igual que la Escuela de Nutrición. Esta situación permite deducir que aunque cada escuela tiene sus propios directivos, los mismos estarían implementando el mismo estilo gerencial, el cual no está propiciando el ambiente idóneo y productivo dentro de las mismas. Además, otro factor que estaría influyendo es la calidad de las relaciones, que en este caso permite deducir que las mismas no son armónicas. 


\section{Tabla 5}

Distribución de las respuestas ULA-Escuela de Medicina. Según las dimensiones estudiadas

\begin{tabular}{|c|c|c|c|c|c|}
\hline Dimensión & $\mathrm{N}$ & Minimo & Máximo & Media & Desviación Estándar \\
\hline $\begin{array}{l}\text { Funcionamiento } \\
\text { Organizacional }\end{array}$ & 53 & 2,00 & 30,00 & 19,2642 & 5,7718 \\
\hline $\begin{array}{l}\text { Estructura } \\
\text { Organizacional }\end{array}$ & 53 & 6,00 & 30.00 & 16,8302 & 5,0602 \\
\hline $\begin{array}{l}\text { Proceso de Toma de } \\
\text { Decisiones }\end{array}$ & 53 & 1,00 & 10,00 & 4,7547 & 2,3608 \\
\hline Tipo de comunicación & 53 & 4,00 & 20,00 & 12,0943 & 4,0109 \\
\hline Motivación al Logro & 53 & 6.00 & 30,00 & 23.5660 & 4,6679 \\
\hline Praxis Educativa & 53 & 8,00 & 40,00 & 31,3396 & 5,0837 \\
\hline
\end{tabular}

Fuente: Encuesta realizada para este trabajo.

N: Tamaño de la muestra.

Tabla 6

ULA-Escuela de Medicina. Distribución de respuestas según las variables estudiadas

\begin{tabular}{lccccc}
\hline Variable & N & Minimo & Máximo & Media & Desviación Estándar \\
\hline Clima Organizacional & 53 & 23,00 & 113,00 & 76,5094 & 17,7359 \\
Desempeño Docente & 53 & 11,00 & 40,00 & 32,1887 & 6,2881 \\
\hline
\end{tabular}

Fuente: Encuesta realizada para este trabajo.

N: Tamaño de la muestra.

De igual manera sobresale la estructura organizacional en lo referente a la existencia de cierto número de niveles jerárquicos que estarían perjudicando el proceso de toma de decisiones, lo que afectaría la transmisión de información entre un nivel y otro (Consejo de Escuela a los Departamentos y cátedras). No obstante, cabe destacar que la Motivación al Logro y la variable Desempeño Docente si presentan valores que lo sitúan como Excelente. Sin embargo, llama la atención que en esta última variable, los docentes de esta escuela, valoran de forma muy favorable su propio desempeño, en cuanto a la responsabilidad y su rendimiento laboral.

Lo anteriormente descrito permite afirmar que los docentes de la Escuela de Medicina, tienen características muy particulares que los diferencian de los demás docentes de la Facultad de Medicina, tales como la motivación al logro y su desempeño, las cuales, probablemente, estarían influenciadas por el status social que tienen, ya que por su título académico gozan de amplios beneficios socioeconómicos y altas posibilidades de alcanzar una trayectoria significativa dentro de la misma universidad y en otras instituciones privadas; situación distinta a la que tienen los docentes de las escuelas de Nutrición y Enfermería. 
También la escuela de Enfermería presenta similares resultados (Tablas 7 y 8 ). Con relación a la Variable Clima Organizacionl, cabe destacar que su valoración se ubica en la categoría regular, de igual manera las dimensiones: Funcionamiento Organizacional, Estructura Organizacional, Proceso de Toma de Decisiones y Motivación al Logro se ubican en esa categoría. Esto evidencia que la percepción del clima organizacional de estos docentes estaría relacionado con el nivel de satisfacción de sus necesidades sociales, reflejando que el mismo no es optimo.

Tabla 7

ULA-Escuela de Enfermeria Distribución de las respuestas según las dimensiones estudiadas

\begin{tabular}{lccccc}
\hline Dimensión & $\mathrm{N}$ & Minimo & Máximo & Media & Desviación Estándar \\
\hline $\begin{array}{l}\text { Funcionamiento } \\
\text { Organizacional }\end{array}$ & 19 & 6,00 & 25,00 & 15,7368 & 5,8104 \\
$\begin{array}{l}\text { Estructura } \\
\text { Organizacional }\end{array}$ & 19 & 6,00 & 26,00 & 16,5263 & 6,1316 \\
$\begin{array}{l}\text { Proceso de Toma de } \\
\begin{array}{l}\text { Decisiones } \\
\text { Tipo de comunicación }\end{array}\end{array}$ & 19 & 2,00 & 8,00 & 5,1579 & 2,3157 \\
$\begin{array}{l}\text { Motivación al Logro } \\
\text { Praxis Educativa }\end{array}$ & 19 & 19 & 4,00 & 18,00 & 11,4211 \\
\hline
\end{tabular}

Fuente: Encuesta realizada para este trabajo.

$\mathrm{N}$ : Tamaño de la muestra.

Tabla 8

ULA-Escuela de Enfermeria

Distribución de respuestas según las variables estudiadas

\begin{tabular}{lccccc}
\hline Variables & N & Minimo & Máximo & Media & Desviación Estándar \\
Clima Organizacional & 19 & 33,00 & 104,00 & 70,0000 & 20,1990 \\
Desempeño Docente & 19 & 18,00 & 36,00 & 31,3158 & 4,2824 \\
\hline
\end{tabular}

Fuente: Encuesta realizada para este trabajo.

$\mathrm{N}$ : Tamano de la muestra.

No así la variable Desempeño Docente y el Tipo de comunicación cuyos valores la sitúan en la categoría de Excelente, lo que indica que los mismos valoran su propia actuación con alta estima y esto les estaría generando satisfacción por la labor cumplida. Sin embargo, puede considerarse esta apreciación como algo intrínseco de estos docentes, los cuales son profesionales de la Enfermería y tienen una connotación de servicio, entrega y vocación que envuelve a esta profesión.

El Análisis de la varianza realizado para comparar cada una de las dimensiones y variables entre las diferentes escuelas, indica que en todos los casos excepto para la dimensión Funcionamiento Organizacional (DFO) no existen diferencias significativas entre 
las tres escuelas (Tabla 9).

\section{Tabla 9}

ULA-Facultad de Medicina. Análisis de la varianza de las respuestas sobre el funcionamiento organizacional

\begin{tabular}{lccccccc}
\hline $\begin{array}{l}\text { Funcionamionto } \\
\text { Organizacional }\end{array}$ & $\mathrm{N}$ & Media & $\begin{array}{c}\text { Desviación } \\
\text { Estándar }\end{array}$ & $\begin{array}{c}95 \% \text { Intervalos de } \\
\text { confianza para la media } \\
\text { L, Inferior }\end{array}$ & L. Superior & Minimo & Máximo \\
\hline Nutrición & 14 & 17,7 & 6,2441 & 14,1090 & 21,3195 & 8,00 & 27,00 \\
Medicina & 53 & 19,3 & 5,7718 & 17,6733 & 20,8551 & 2,00 & 30,00 \\
Enfermeria & 19 & 15,7 & 5,8104 & 12,9363 & 18,5373 & 6,00 & 25,00 \\
\hline
\end{tabular}

Fuente: Encuesta para este trabajo.

N: Taman̄o de la muestra.

El p-value obtenido en la tabla del análisis de la varianza dio 0.08 , lo que significa que existen diferencias significativas entre las tres escuelas cuando se comparan con respecto a la dimensión Funcionamiento Organizacional.

Con el fin de detectar esas diferencias se realizó un análisis a porteriori aplicando la prueba Least Significand Difference (Diferencia Mínima Significativa) (Tabla 10).

Tabla 10

ULA-Facultad de Medicina.

Prueba diferencia minima significativa de las respuesta de los docentes sobre el funcionamiento organizacional

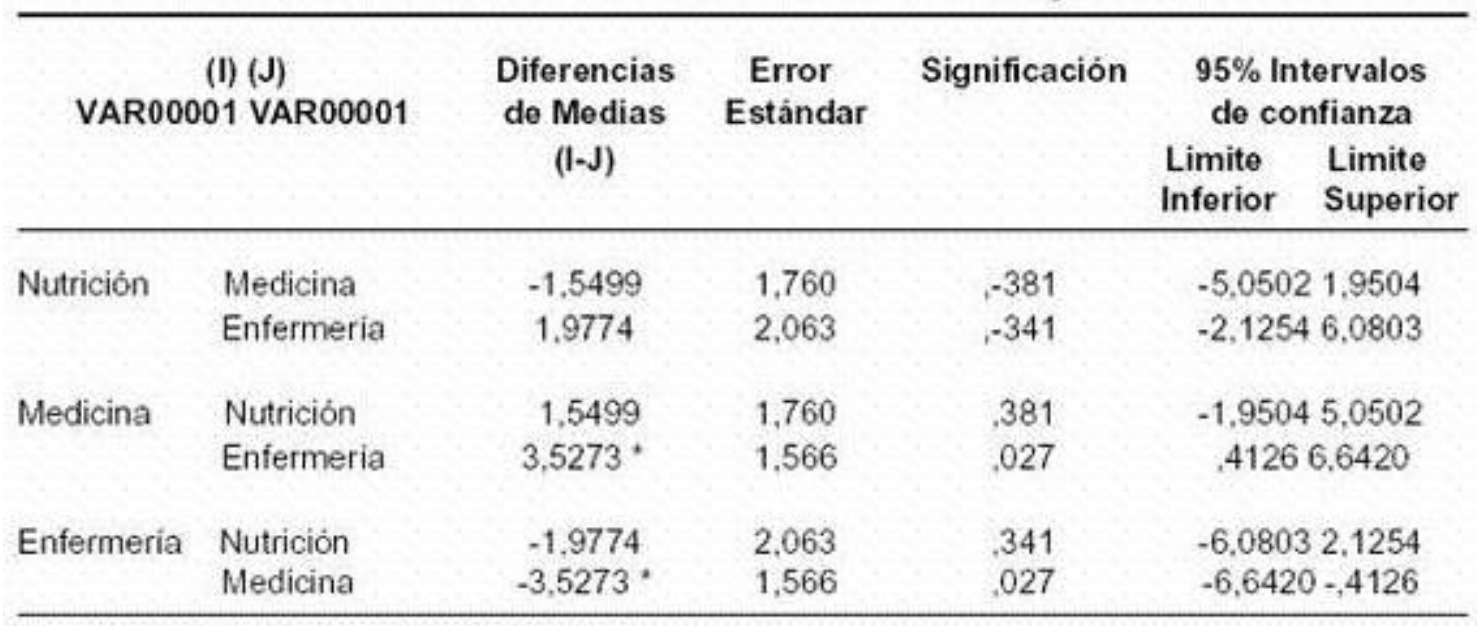

*las diferencias de medias es significativa al $5 \%$.

Fuente: Encuesta realizada para este trabaio.

\section{Conclusiones}

Los resultados obtenidos reflejan que los docentes de las escuelas de Medicina y Enfermería presentan una valoración similar con respecto al Funcionamiento 
Organizacional, el cual consideran inadecuado, siendo una de las razones de esta situación la estructura existente, que no estaría favoreciendo la eficiencia de la facultad. Sin embargo, ambas apreciaciones son diferentes a la de la escuela de Nutrición, que presenta un valor promedio más bajo. Esto podría estar relacionado con el estilo gerencial, la calidad de la supervisión y las relaciones interpersonales existentes en la misma, lo que estaría generando un clima ńgido y poco productivo que influye en el comportamiento de los miembros del personal profesoral.

De los mismos, también se destacan algunas debilidades en cuanto al funcionamiento organizacional en lo referente al estilo gerencial predominante, el proceso de toma de decisiones concentrado en los directivos lo que genera que la información no baje a los escalones más bajos y que sea poco difundida en las cátedras, propiciando que la relaciones interpersonales no sean armónicas y haya poca cooperación entre sus miembros. Además, la existencia de cierto número de niveles jerárquicos, muchas veces le confiere rigidez a la estructura organizacional, ya que por la misma, estar basada en el modelo piramidal; ha conducido a engendrar un clima cerrado y controlado, que repercute en el comportamiento organizacional de sus miembros y en su rendimiento académico.

No obstante, el Análisis de la Varianza realizado para comparar cada una de las dimensiones y variables entre las diferentes escuelas, indica que en todos los casos excepto para la dimensión Funcionamiento Organizacional no existen diferencias significativas entre las tres escuelas.

\section{Propuesta de Gestión para optimizar el clima organizacional y el desempeño de los docentes de la Facultad de Medicina}

Los contenidos de esta propuesta de gestión, pretenden instrumentar acciones que permitan orientar, mejorar y modernizar los procesos gerenciales y de toma de decisiones, así como aumentar la flexibilidad y eficiencia de los mismos. También, se busca optimizar el desempeño académico de los profesores adscritos a esta dependencia, de tal forma que puedan desarrollar habilidades y destrezas en su labor profesional, contribuyendo de esta manera a elevar la calidad de la formación de los futuros profesionales de la salud. Por ello, esta propuesta estará dirigida al personal docente, directivo (Directores, Jefes de Departamentos y Jefes de Cátedras) y miembros del Consejo de la Facultad de Medicina.

De esta manera se espera que se transforme esta facultad en una organización con carácter dinámico, que se movilice en función de las metas y que le proporcione 
satisfacción laboral a los docentes, lo cual incidiría en el ambiente general de la organización, produciendo un clima de armonía y estabilidad psicológica, tan importante en la labor académica. Además, se ha detectado en el personal directivo la necesidad de adquirir conocimientos sobre el proceso gerencial, a objeto de llevar a la práctica las diferentes funciones administrativas con plena eficiencia y dinamismo, coordinando con precisión, pero con flexibilidad, la participación de todos los actores de la comunidad universitaria y así controlar el funcionamiento organizacional de su respectiva escuela.

Asimismo, esto permitirá optimizar las funciones gerenciales de los docentes, cuyo propósito contribuirá a desarrollar sus destrezas y habilidades y así mejorar su labor profesional, elevando la calidad de la educación universitaria de nuestro país.

Por medio de la administración de la propuesta se busca lograr el cumplimiento de los objetivos trazados para lo cual se requiere de un ambiente apropiado que facilite el logro de los resultados. En consecuencia el factor humano es importante porque a través de el se cumplen las actividades, en un proceso de integración de recursos para lograr objetivos y alcanzar metas preestablecidas en la forma más eficiente con la participación de los docentes de la facultad. Por ello esta propuesta de gestión será administrada por las autoridades de las respectivas escuelas en coordinación con los especialistas del Centro de Investigación y Desarrollo Empresarial de la Facultad de Ciencias Económicas (CIDE) de la ULA. En este sentido, la administración de la propuesta de gestión se elaboró con base a las personas que van a desempeñar las funciones de facilitadores y realizarán las actividades previstas dentro de los lapsos estipulados.

\section{Factibilidad I nstituciona}

Al respecto, cabe destacar que cumplida la etapa inicial de revisión y análisis de la razones de la problemática universitaria actual de la Universidad de Los Andes, por decisión del Consejo Universitario, se ha convocado a todas las instancias existentes en la misma(miembros de la comunidad universitaria) a participar colectivamente en las mesas de discusión a fin de proponer estrategias relacionadas con la transformación, en cuanto a la Misión de la universidad, la carrera académica de profesores y estudiantes, la estructura universitaria-gobierno y gestión y la relación de la universidad con el entorno. Por ello se considera que esta propuesta responde a este planteamiento de cambio y transformación de la gestión académica de los docentes.

\section{Factibilidad Técnica}


En cuanto a los recursos humanos que se tomarán en cuenta para la implantación de la propuesta, se debe señalar que los facilitadores serán dos profesores expertos del Centro de Investigaciones Empresariales de la Facultad de Ciencias Económicas y Sociales, quienes cumplen estas funciones de capacitación en el mismo. Esta participación podría ser cubierta con el aporte financiero del Vicerrectorado Académico de la U.L.A.

\section{Factibilidad Financiera}

Se ha determinado que esta será posible a través del apoyo de la gestión de los directores de las escuelas de la Facultad de Medicina y el aporte financiero del Vicerrectorado Académico, quien tiene la responsabilidad de desarrollar estrategias que permitan la actualización y capacitación de los docentes universitarios. Por ello, se estima que los gastos derivados de los recursos materiales a utilizar, serán cubiertos por estas dependencias.

* El presente artículo es resultado de un proyecto de investigación financiado por el CDCHT de la ULA.

\section{Referencias Bibliográficas}

1. Dessler, Gary (1991), Organización y Administración: Enfoque Situacional. Prentice Hall Interamericana. México

2. Feliú, Pedro (2000), Evaluación organizacional aumenta la productividad de las compañías. Diario EL NACIONAL. E/10. 20 Febrero 2000. Caracas. Venezuela.

3. Goncalves, Antonio (1999), Dimensiones del Clima Organizacional. http: www. Calidad.org./articles/dec97/2de97. htm. Consulta: 23-10-1999.

4. López, Antonio; Saturno, Pedro; Urrea, Angel; García, Manuel; Ballesteros, Alejandro y Sáez, Celina (1999), El clima laboral en los Profesionales de Atención Primaria, Cartagena Colombia. http: www. graduado.com/tesis Consulta: 15-05-1999.

5. Menárguez, Juan y Saturno, Pedro (1999), Descripción del clima organizacional en equipos de atención primaria de una comunidad autónoma. CUIDEN. Base de Datos Bibliográficas de la Fundación Index. Atención Primaria. mar; 23 (5):280-284. Madrid.

6. Pérez, Reyna y Sanabria, Alicia (1997), El Clima organizacional en el Decanato de Ingeniería Agronómica de la Universidad Centrooccidental Lisandro Alvarado. Venezuela. 
Revista Investigación y Postgrado. Vol.12, N 1, Caracas.Venezuela. Universidad Centrooccidental Lisandro Alvarado. Vicerrectorado de Investigación y Postgrado, pp. 5662.

7. Stoner, Joseth; Hughes, Charles y Tight, Marlon (1996), Administración. $6^{\circ}$ Edición. Pearson Educación Editorial Prentice-Hall Hispanoamericana. México.

8. Solla, Carmen (1998), “El clima organizacional y su relación con la calidad de servicio prestado por el Colegio Universitario de Administración y Mercadeo. Extensión Puerto Cabello, durante el Semestre 96-B." Tesis de Maestría. Universidad Bicentenaria de Aragua.

9. Universidad de Los Andes (1995), Boletín de la Estructura Organizativa de la Universidad de Los Andes. Oficina de Información, Estadística y Control. Secretaría de la Universidad de Los Andes. Mérida. Venezuela.

10. Universidad de Los Andes (2001), Informes de las Mesas de Trabajo. Comisión Operativa para el Cambio. Secretaría de la ULA. Mérida, Venezuela.

11. Universidad Pedagógica Experimental Libertador (1996), Reglamento de Elaboración de Tesis de Grado. Caracas, Venezuela.

12. Universidad Pedagógica Experimental Libertador (1998), Instituto de Mejoramiento Profesional del Magisterio. Curso de Investigación Educativa. Caracas, Venezuela. 